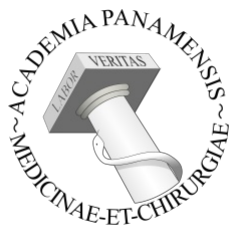

\title{
Artículos originales \\ Dosis Diaria Definida de antibióticos en pacientes atendidos en hospitales públicos de Panamá
} [Defined Daily Dose of antibiotics in patients treated in public hospitals in Panama]

Eric Ivan Conte ${ }^{1}$, Cecilio Niño1, Cesar Zamorano², Yovany Morales², Julio Toro², Beatriz Gómez³, ${ }^{1}$ Instituto Conmemorativo Gorgas de Estudios de la Salud, Panamá, Panamá;²Caja de Seguro Social, Panamá; 3Universidad Latina de Panamá. Panamá;

\section{Palabras Claves: \\ antibióticos, Dosis Diaria Defini- da,consumo, prescripción, costo \\ Keywords: antibiotics, Defined Daily Dose, con- sumption, prescription cost. \\ Correspondencia a: \\ Eric Ivan Conte \\ Correo electrónico: econte@gorgas.gob.pa \\ Recibido: \\ 22 de sep de 2021 \\ Publicado: \\ 24 de diciembre de 2021 \\ Aspectos bioéticos: \\ Los autores declaran que no existe conflicto de interés y que el trabajo fue aprobado por el Comité institucio- nal de ética del CHDrAAM.}

Financiamiento: Los autores declaran que no hubo financiamiento externo para la realización de este trabajo, y que la información cruda se compartirá a solicitud.

\section{Uso y reproducción:}

Publicación de libre uso individual, no comercial. Prohibida la distribución para otros usos sin el consentimiento escrito del editorial.

\section{Resumen}

Objetivo: Determinar la Dosis Diaria Definida (DDD) de antibióticos utilizados en los Servicios de Medicina Interna de cuatro hospitales públicos en Panamá. Métodos: Se escogió una muestra a conveniencia de 4 hospitales públicos de segundo nivel de atención de la seguridad social y Ministerio de Salud, atendiendo características en cuanto a ubicación en provincias, población atendida, servicios médicos ofrecidos y con Dosis Unitaria en funcionamiento. Se obtuvieron datos de los pacientes hospitalizados entre los meses de enero a marzo del 2017 y la información era registrada en una base de datos anotándose generales del paciente, diagnósticos, antibióticos, dosis, frecuencia, tiempo de tratamiento. Igualmente se anotaron los consumos y costos unitarios de todos los antibióticos usados en los hospitales durante el año 2016, para calcular la DDD y Dosis Diaria Prescrita. Resultados: La oxacilina fue el antibiótico con mayor DDD días cama con 60.5, luego le siguen ceftriaxona (45.2), cefalotina (35.5), clindamicina (35.5). Por grupo farmacológico, las cefalosporinas tienen consumos más altos con 87.6 DDD días cama, la penicilina MRSA 60.5, la lincosamidas con 35.5 DDD días cama, seguido de las quinolonas con 29.3. Conclusiones: Los resultados pueden ser utilizados para implementar programas para mejorar el uso racional y gasto de antibióticos a nivel hospitalario y que la DDD se use para medir el consumo y a la vez propiciar el control de antibióticos a través de comunicaciones escritas con los médicos tratantes. Se deben realizar estudios de DDD días cama relacionando con los aspectos microbiológicos para relacionar mejor el uso de los antibióticos.

\section{Abstract}

Objective. Determine the Defined Daily Dose of antibiotics used in the Internal Medicine Services of 4 public hospitals in Panama. Methods. A convenience sample of 4 public hospitals of the second level of social security care and the Ministry of Health was chosen, considering characteristics in terms of location in the provinces, population served, medical services offered and with Unit Dose in operation. Data were obtained from hospitalized patients between the months of January to March 2017 and the information was recorded in a database, noting general patient reports, diagnoses, antibiotics, dose, frequency, treatment time. Likewise, the consumptions and unit costs of all antibiotics used in hospitals during 2016 were recorded to calculate the DDD and Prescribed Daily Dose. Results. Oxacillin was the antibiotic with the highest DDD bed days with 60.5 , followed by ceftriaxone (45.2), cephalothin (35.5), and clindamycin (35.5). By pharmacological group, cephalosporins have higher intakes with 87.6 DDD bed days, penicillin MRSA 60.5, and lincosamides with 35.5 DDD bed days, followed by quinolones with 29.3. Conclusions. The results can be used to implement programs to improve the rational use and expense of antibiotics at the hospital level and that the DDD is used to measure the consumption and at the same time promote the control of antibiotics through written communications with the treating physicians. Bedday DDD studies should be carried out relating to microbiological aspects to better relate the use of antibiotics.
Órgano oficial de la Academia Panameña de Medicina y Cirugía y del Instituto Commemorativo Gorgas para Estudios de la Salud (ICGES). Indexado en LILACS, EBSCO, y www.revistasmedicas.org.
R M P

RMDP 2021; 41(3):34-41 


\section{INTRODUCCIÓN}

De acuerdo con la Organización Mundial de la Salud (OMS), el uso racional de medicamentos se define como la acción que permite "que los pacientes reciban la medicación adecuada a sus necesidades clínicas, en las dosis correspondientes a sus requisitos individuales, durante un período adecuado y al menor coste posible para ellos y para la comunidad" [1].

En el caso particular de los antibióticos, un mal uso conlleva al surgimiento de la resistencia a los mismos, la cual se produce cuando los microorganismos sufren cambios al verse expuestos a los antimicrobianos, lo que puede provocar que estos agentes se vuelven ineficaces y las infecciones persisten en el organismo [2].

La falta de un adecuado control de antibióticos en Panamá ha originado el surgimiento y crecimiento de la resistencia bacteria, tal como se encontró en un estudio desarrollado por el Instituto Conmemorativo Gorgas de Estudios de la Salud (ICGES) donde se concluyó que los grupos de antibióticos con mayor resistencia bacteriana fueron los b-lactámicos, las cefalosporinas y las quinolonas. Las bacterias con mayor resistencia a nivel nacional son E. coli, K. pneumoniae, Acinetobacterspp y P. aeruginosa durante el periodo 2007-2013 [3].

El Sistema de Dispensación de Medicamentos por Dosis Unitaria (SDMDU) o también conocido como Unidosis, tiene como objetivo que los medicamentos estén disponible en el lugar apropiado y en forma oportuna para su aplicación al paciente, prescritos por su médico tratante en las dosis y formas farmacéuticas por éste señaladas, deseándose: racionalizar la distribución de medicamentos, la terapéutica farmacológica, disminuir los errores de medicación, procurar la correcta administración de los medicamentos al paciente e integrar al farmacéutico al equipo asistencial en la atención del mismo [4].

En el caso específico de Panamá, de los 16 hospitales de la Caja de Seguro Social (CSS) para el año 2017, 15 tenían SDMDU en funcionamiento, el cual fue implementado inicialmente en el Complejo Hospitalario Dr. Arnulfo Arias Madrid en 1989 con una cobertura inicial de 40 camas en la Sala de Medicina Interna [5]. Posteriormente se fue extendiendo por diversas salas de hospitalización y de ahí emigró hacia otros hospitales institucionales y posteriormente a hospitales privados.

La OMS recomienda aplicar la Clasificación Anatómico Terapéutica (ATC por sus siglas en inglés) para analizar el uso de los medicamentos, de la prescripción, tanto de la cantidad como de la calidad, utilizando diversas unidades, fundamentalmente la Dosis Diaria Definida (DDD), y la Dosis Diaria Definida por mil habitantes y día (DHD) con las que se construyen diferentes indicadores de prescripción [6]. La DDD, es una unidad diferente para cada fármaco, es la dosis media diaria supuesta de un fárma- co, cuando se usa en su indicación principal, es decir, es una unidad técnica internacional de medida del consumo de medicamentos, que se establece de acuerdo con las recomendaciones de las publicaciones científicas y del laboratorio fabricante [7]. Mediante un estudio observacional y de corte transversal, se describió el consumo y las prácticas de prescripción de antibióticos parenterales en el servicio de medicina interna de un hospital de Bogotá, donde se administraron $32.6 \mathrm{DDD} / 100$ pacientes/día, siendo los grupos terapéuticos más prescritos: cefalosporinas, penicilinas y quinolonas [8].

La Dosis Diaria Prescrita (DDP) es definida como el promedio de dosis prescrita de acuerdo con una muestra representativa de prescripciones de medicamentos durante un tiempo definido y de pacientes. La DDP puede ser determinada desde estudios de prescripciones o médicos o de registro de farmacias. Es importante relacionar la DDP con la dosis usual y la misma dará la cantidad promedio diario de un medicamento que es actualmente prescrito [9].En la actualidad, el SDMDU en Panamá, no ejerce un adecuado control sobre el uso final de los antibióticos prescritos en pacientes hospitalizados en los sistemas públicos de salud, lo que en ocasiones pudiera prolongar la estadía intrahospitalaria y por ende los gastos originados en salud.

Así mismo la DDD no se incluye dentro de los procesos desarrollados por el sistema de Dosis Unitaria, por lo que pretendemos calcular la DDD día-cama y establecer si este indicador aplicado en las labores de rutina del farmacéutico serviría en el uso racional de antibióticos, creando herramientas que puedan ser usadas para evitar un uso irracional. Considerando lo anterior nos proponemos estudiar la utilización y uso racional de antibióticos en pacientes hospitalizados en los Servicios de Medicina Interna en algunos hospitales públicos ubicados en diferentes provincias en la República de Panamá.

A pesar del tiempo transcurrido desde la recolección de los datos y la tabulación de los resultados vemos pertinente la publicación de los resultados de este estudio pues la información obtenida debe ser valiosa herramienta de trabajo para el desarrollo de las actividades del farmacéutico hospitalario y que este sistema de medida de consumo de medicamentos sea adoptados por los hospitales públicos de nuestro país.

\section{MATERIALES Y MÉTODOS}

Se trata de un estudio de corte transversal, retrospectivo donde se seleccionó una muestra a conveniencia de 4 hospitales públicos de segundo nivel de atención del Ministerio de Salud (MINSA) y de la Caja de Seguro Social (CSS) de Panamá, atendiendo características particulares en cuanto a ubicación en diversas provincias, población atendida, servicios médicos ofrecidos y desarrollo de los servicios de farmacia. En la ciudad de Panamá se incluyó el Hospital Susana Jones (HSJ) de la CSS y el Hospital San Miguel Arcángel (HSMA) del MINSA. En Ve- 
Eric Ivan Conte et.al. - Dosis Diaria Definida de antibióticos en pacientes

raguas, el Hospital Luis Fábrega (HLF) del MINSA y en Chiriquí, el Hospital Regional Rafael Hernández (HRRH) de la CSS.

Unidad de observación: Los perfiles farmacoterapéuticos de los pacientes hospitalizados en las salas de medicina interna de los cuatro hospitales durante los meses de enero, febrero y marzo del año 2017. El tamaño de la muestra comprendió a los pacientes que estuvieron hospitalizados durante ese periodo. Los consumos y costo unitario de los antibióticos empleados en los diversos hospitales durante el año 2016 también fueron considerados.Los hospitales del estudio eran de segundo nivel de complejidad, que contaran SDMDU, con el uso de perfiles farmacoterapéuticos de los pacientes hospitalizados en las salas de medicina y que recibieron antibióticos con o sin otros medicamentos.

\section{Procedimiento para la recolección de datos}

Los farmacéuticos ingresaron los datos desde los perfiles farmacoterapéuticos usados en el SDMDU de pacientes que hayan sido dado de alta o egresados de los hospitales del estudio durante los meses de enero a marzo de 2017.

Para la recolección de la información desde los perfiles se utilizó una base de datos que fue instalada en computadoras de las farmacias donde se realizó el estudio.

Cada perfil farmacoterapéutico se pasó a un formato electrónico de recolección de datos el cual fue codificado y se anotó la edad, sexo, diagnósticos, servicio médico y alergias, sin anotar nombre ni cedula de identificación ni seguro social del paciente. En cada perfil se anotaron los antibióticos con su nombre, presentación, forma farmacéutica, dosis, frecuencia de administración y tiempo de tratamiento recibido por el paciente. Se anotaron otros medicamentos que se administraron concomitantemente con los antibióticos. Se procedió a realizar un cálculo de Dosis Diaria Definida días/cama de acuerdo a formula internacionalmente definida y de Dosis Diaria Prescrita. Se obtuvieron los consumos y costo unitario de los antibióticos empleados en los hospitales durante el año 2016. Los investigadores del ICGES mantuvieron constante comunicación y coordinación con los farmacéuticos de los hospitales y la información recopilada fue remitida el ICGES para los análisis del caso.

\section{Plan de Análisis de los Resultados}

La fórmula matemática de aplicación incluyó: nombre del medicamento, la denominación común internacional, la presentación farmacéutica (ampollas, vial etc.), el contenido de principio activo (mg, g, unidades) y la cantidad dispensada. Se realizó la clasificación de los antimicrobianos según la clasificación ATC y la asignación de la DDD, usando el motor especializado de búsqueda en línea; WHO Collaborating Centre for Drug Statistics Methodology. El consumo hospitalario, expresado por lo general en unidades dispensadas, se convirtió a gramos a partir del número de unidades administradas de un mismo fármaco.

Con la DDD recomendada por la OMS y las estadísticas hospitalarias (número de camas y porcentaje de ocupación) se puede obtener el consumo en DDD/100 camas-día.
El cálculo se realizó según la siguiente fórmula [10]:

DDD/100 camas-día $=a \times 100$

\section{$\mathrm{b} \times \mathrm{t} \times \mathrm{n} \times \mathrm{Oc}$}

donde:

a: consumo en gramos del antibacteriano usado.

b: DDD recomendada del fármaco por la OMS en el período analizado, gramos.

t: tiempo analizado, en días.

$\mathrm{n}$ : número de camas del hospital.

Oc: porcentaje de ocupación de camas hospitalarias.

Para el cálculo de Dosis Diaria Prescrita se usó la siguiente fórmula:

\section{Gramos del Antibiótico Consumido Número de pacientes con el Agente}

Para el cálculo de Costo de DDD se utilizó la siguiente fórmula [11]:

$$
\text { Costo/DDD }=\frac{\text { DDD } \times \text { CU }}{\text { CDU }}
$$

DDD: Dosis Diaria Definida por OMS

CU: $\quad$ Costo por Unidad (equivalente al dólar americano) CDU: Contenido del principio Activo por Dosis Unitaria (miligramos o gramos)

\section{Programas a utilizar para análisis de datos}

El programa de captura de datos utilizado para registrar la historia de medicamento del paciente, fue el software CSPro versión 6.3 creado por la Oficina de Censos de los Estados Unidos, adicionalmente se desarrolló un diccionario de datos con cada una de las variables que luego de ser registrados mediante la pantalla de captura fueron almacenados para análisis de datos mediante el SPSS versión 24. Para la confección de gráficas se utilizó Microsoft Excel.

\section{Aspecto Éticos}

El estudio fue sometido para su aprobación a la Coordinación en Regulación de Investigación para Salud del Ministerio de Salud, a la Sección de Investigación y Bioética de la Caja de Seguro Social y al Comité de Bioética de la Investigación del ICGES que lo autorizó mediante Nota No. 516/CBI/ICGES/17 de 2 de junio de 2017. Se accedió a los perfiles farmacoterapéuticos durante el periodo de estudio y en ninguna circunstancia se anotaron nombres ni números de identificación como número de cédula o número de seguro social de los perfiles farmacoterapéuticos de los pacientes. La privacidad del paciente fue conservada y nunca se expuso su nombre, ni se asoció el paciente con los antibióticos usados. No fue un estudio donde se accedió a expedientes clínicos de los pacientes atendidos ni tampoco hubo contacto con ellos. Tampoco se incluyó los nombres de médicos y farmacéuticos que prescribían y dispensaban los medicamentos. 


\section{RESULTADOS}

Los cuatro hospitales que participaron en el estudio aportaron un total de 461 pacientes, 256 eran del sexo femenino $(55.5 \%)$ y 205 del sexo masculino (44.5\%). El HLF participo con 70 pacientes, el HRRH 208, el HSMA con 144 y el HSJ con 39 pacientes durante los meses de enero, febrero y marzo del año 2017 (Gráfica No1).

La edad promedio de los pacientes fue de 62 años para los hospitalizados en el HLF, 51 años para el HRRH, 66 años para el HSMA y 78 años para el HSJ. La media en edad fue 64.5 años.

Las enfermedades del sistema cardiocirculatorio de acuerdo a la Clasificación Internacional de Enfermedades fueron las más comunes con un $28 \%$, seguido del sistema respiratorio con $21 \%$ y enfermedades infecciosas con un $16 \%$, tal como se aprecia en la Gráfica No. 2.

En la Gráfica No. 3 se observa la Dosis Diaria Definida (DDD) recomendada por la OMS que es la dosis media diaria supuesta de un fármaco, cuando se usa en su indicación principal. La ceftriaxona (CRO) marcó 11.7 DDD días hospital en el HLF y la DDD de la OMS es de 2 DDD. La clindamicina marcó 14.8 vs 1.8 de OMS y la oxacilina fue de 22.1 comparado con 2 de OMS. La amikacina (AMK), el meropenem (MEM) y la vancomicina marcaron niveles parecidos a los de la OMS.

Los valores obtenidos de las DDD días camas del HRRH de los renglones CRO, metronidazol (MET), oxacilina La Dosis Diaria Prescrita (DDP) desarrollada durante el (OXA) resultaron muy superiores a las DDD recomenda- tiempo del estudio en 3 meses del año 2017 fue aplicada das por OMS. La AMK, imipenem/cilastatin (IPM) y van- para los pacientes de las salas de medicina, en este caso comicina (VAN) resultaron en el mismo nivel, mientras del HSJ (Gráfica No. 6), donde la OXA marcó el valor que la cefotaxima (CTX) y ceftazidima (CAZ) presentan más alto de DDP, seguido de meropenem.

Gráfica 4. Comparación de Dosis Diaria Definida de Antibióticos usados en el Hospital Luis Fábrega, 2016.

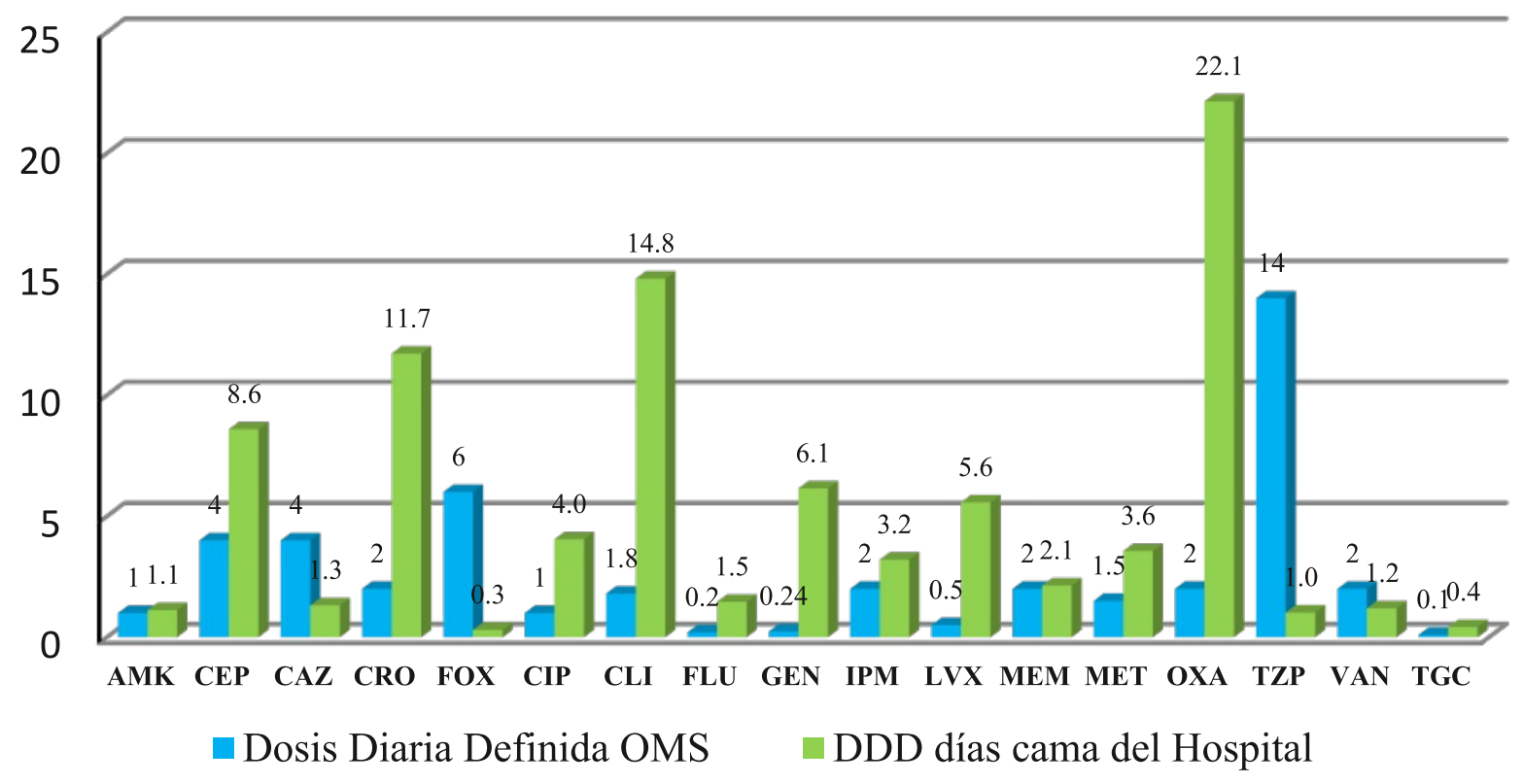


Gráfica 4. Comparación de Dosis Diaria Definida de Antibióticos usados en el Hospital Regional Rafael Hernández, 2016.

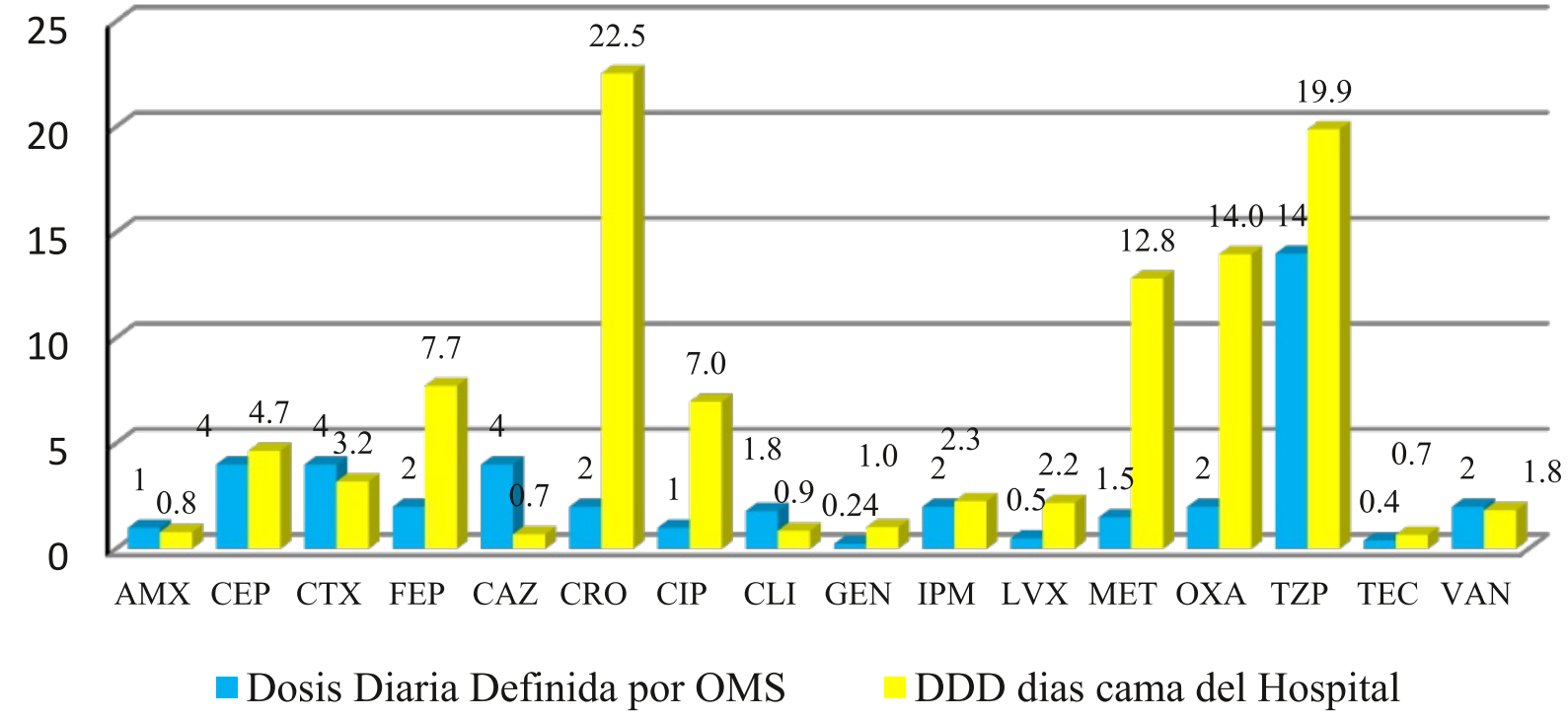

Gráfica 5. Comparación de Dosis Diaria Definida de Antibióticos usados en el Hospital Susana Jones, 2016.

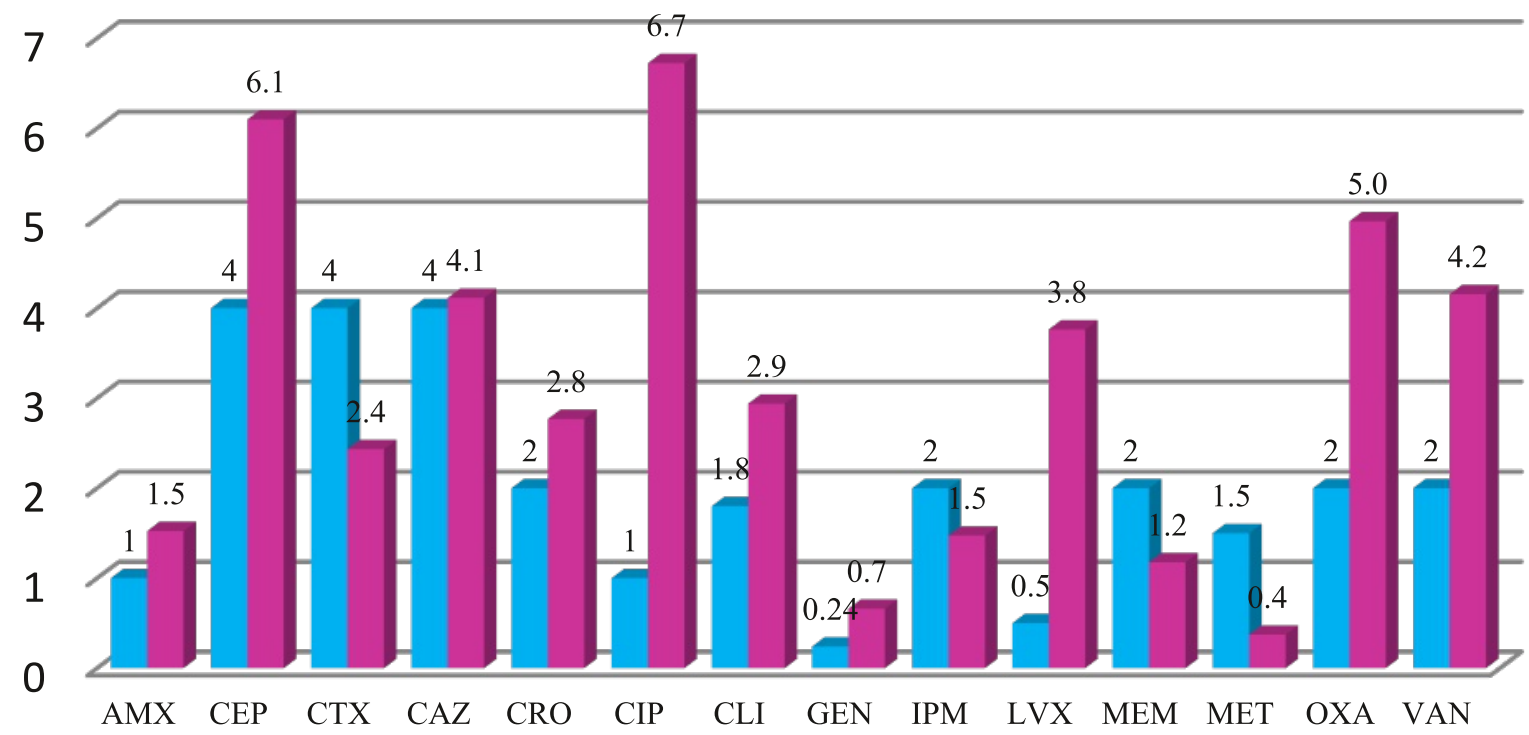

- Dosis Diaria Definida por OMS

- DDD dias cama del Estudio

La DDP para el HSMA se muestra en la Gráfica No. 7, con valor tres veces más elevado cuando vemos la piperacilina/tazobactamcomparada con valores de OMS; los otros antibióticos muestran valores parecidos a OMS.

Cuando totalizamos las DDD días cama para los 4 hospitales de acuerdo a los consumos registrados por las farmacias durante el año de 2016, la OXA fue el antibiótico con mayor DDD días cama con 60.5 , esto significa que por cada 100 días cama en el año, 60 pacientes han consumido 1 gramo del antibiótico diariamente. Luego le siguen CRO (45.2), cefalotina (35.5) y clindamicina (35.5). Los antibióticos que presentaron la DDD más baja fueron: la AMK (3.6), CAZ (6.9), vancomicina (7.6).
Por grupo farmacológico, las cefalosporinas tienen consumos más altos con 87.6 DDD días cama, la penicilina (MRSA, stafilococos aureus resistente a la meticilina) con 60.5 , la lincosamidas con 35.5 DDD días cama, las quinoIonas con 29.3 días cama. El Estudio sobre resistencia bacteriana en Panamá en el 2015 del ICGES mostró que las familias de antibióticos más afectados por la resistencia bacteriana fueron los b-lactámicos, cefalosporinas y quinolonas [4], es decir, que los resultados ya vistos de ese año van de acorde con los resultados de las DDD más alta para los mismos grupos farmacológicos.

La DDP calculada en los meses de enero a marzo de 2017 desde perfiles farmacoterapéuticos presentó por 
Gráfico 6. Comparación de DDD OMS y DDP en el Hospital Susan Jones, 2017.

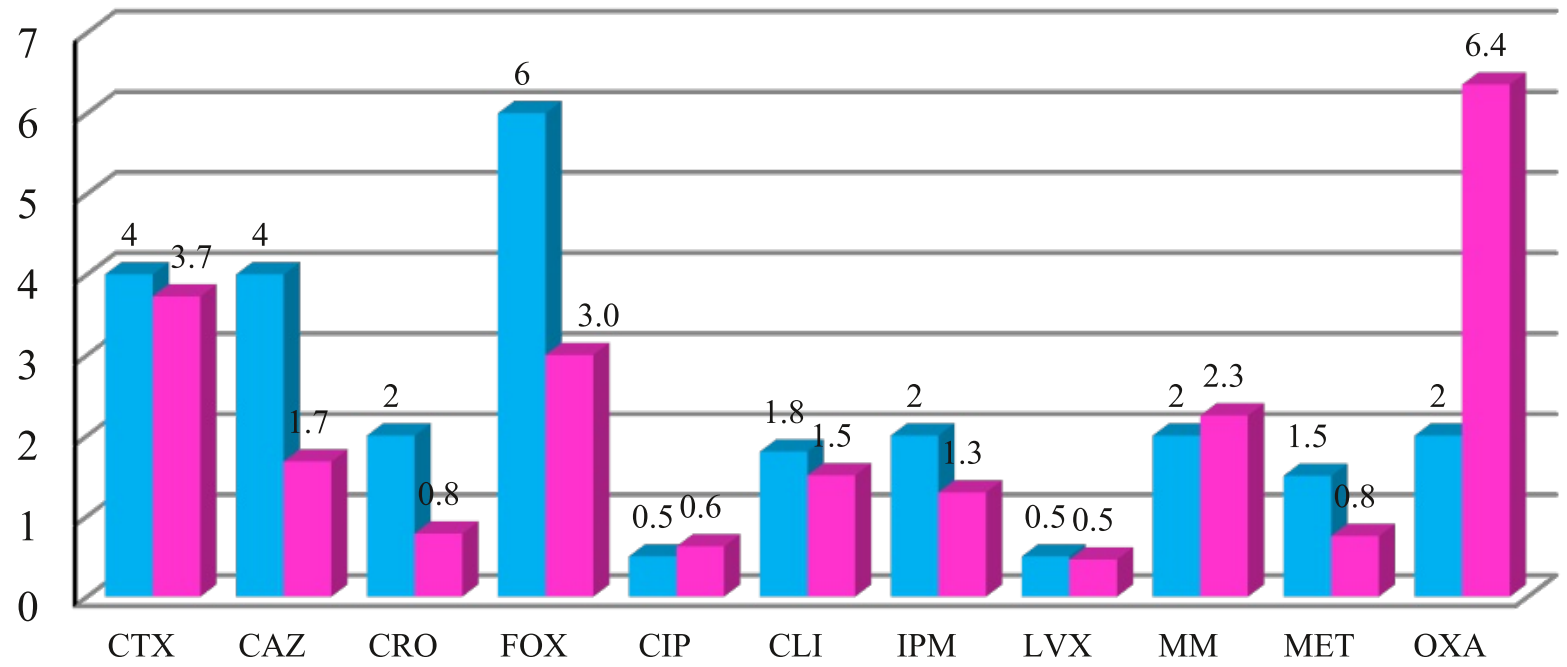

- Dosis Diaria Definida OMS $\quad$ Dosis Diaria Prescrita

Gráfica 7. Comparación de DDD OMS y DDP en el Hospital San Miguel Arcángel, 2017.

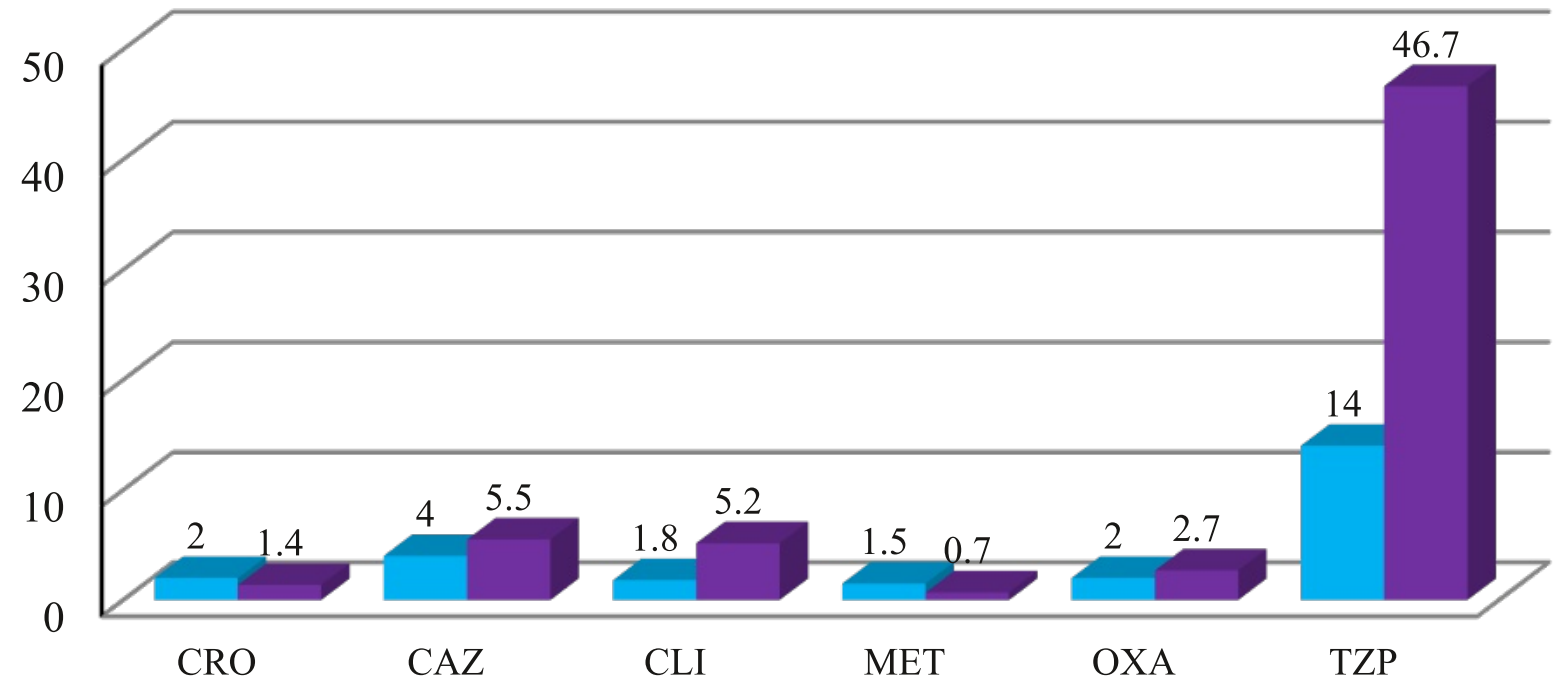

Dosis Diaria Definida OMS — Dosis Diaria Prescrita

Cuadro 1. Comparación Costo Unitario VS Costo/DDD de 5 Antibióticos en los Hospitales del Estudio durante 2016.

\begin{tabular}{lcccccccc} 
& \multicolumn{2}{c}{$\begin{array}{c}\text { Hospital Luis } \\
\text { Fábrega }\end{array}$} & \multicolumn{2}{c}{$\begin{array}{c}\text { Hospital Rafael } \\
\text { Hernández }\end{array}$} & \multicolumn{2}{c}{$\begin{array}{c}\text { Hospital Susana } \\
\text { Jones }\end{array}$} & \multicolumn{2}{c}{$\begin{array}{c}\text { Hospital San Miguel } \\
\text { Arcángel }\end{array}$} \\
\hline & $(\$)$ & Costo/DDD & $(\$)$ & Costo/DDD & $(\$)$ & Costo/DDD & (\$) & Costo/DDD \\
Amikacina 0.5g/2ml & 0.5 & 1 & 1.66 & 2.65 & 1.27 & 2.54 & 0.29 & 0.58 \\
Ceftriaxone 1g vial & 0.4 & 0.8 & 0.56 & 12.63 & 0.33 & 0.66 & 0.36 & 0.72 \\
Oxacilina 1g vial & 0.3 & 0.3 & 0.37 & 5.37 & 0.24 & 0.48 & 0.27 & 0.54 \\
Metronidazol 0.5g/vial & 0.87 & 22.3 & 0.87 & 22.3 & 0.41 & 1.23 & 0.38 & 1.15 \\
Vancomicina 0.5g vial & 3.5 & 14 & 3.33 & 13.32 & 4.68 & 18.72 & 4.49 & 17.96 \\
\hline
\end{tabular}


grupo farmacológico los siguientes valores: 90.1 para los Carbapenémicos, las cefalosporinas con 33.6, penicilina MRSA 32.8. Estos grupos son los mismos que marcan los valores más altos de DDD en los hospitales del estudio durante el año 2016.

En el Cuadro No. 1 se seleccionaron cinco antibióticos con el costo unitario anual en dólar de cada uno de los hospitales para luego comparar con los Costos/DDD, el cual se vio incrementado globalmente en un $64 \%$.

Si analizamos el costo unitario por cada hospital, vemos como la ampolla de amikacina de $500 \mathrm{mg} / 2 \mathrm{ml}$ varía entre 0.29 de dólar en el HSMA hasta \$ 1.66 en el HRRH. En el caso de la Vancomicina $500 \mathrm{mg}$ vial en el HRRH fue de \$ 3.33 y en el HSJ fue de $\$ 4.68$. Estos ejemplos nos indican desigualdad de precios en los hospitales del país para la adquisición de estos agentes.

\section{DISCUSIÓN}

En un estudio en Perú en un hospital de 255 camas de segundo nivel, se encontró que la DDD/100 fueron de ceftriaxona 9.31, ceftazidima 6.27 e imipenem/cilastatin 4.39 [12]. La ceftriaxona registró 11.7 DDD días en el HLF con 235 camas, la ceftazidima con 1.32 y el Imipenem/Cilastatin marcó 3.20 DDD/100 días cama. En el HSMA de 250 camas, para la ceftriaxona se obtuvo un valor de 8.23, ceftazidima 0.76 y cuando observamos las cifras del HRH (304 camas) se obtuvieron los siguientes valores: ceftriaxona 22.55 , ceftazidima 0.70 , imipenem cilastatin 2.26.

En un hospital de alta complejidad de la región metropolitana de Chile se realizó un estudio sobre consumo de antibióticos de uso restringido en dos periodos de tiempo de un año cada uno y se pudo observar un consumo de DDD/100 camas/día de 4.8 para la vancomicina [11] y para lo observado en Panamá para el mismo antibiótico fue de $4.2 \mathrm{DDD} /$ días cama en el HSJ, 2 DDD/días en el HRRH y de 1.2 en el HLF.

En estudio realizado en un hospital del Ecuador en el 2015 [13] se analizaron el consumo promedio de todos los antibióticos y se determinó que los fármacos más consumidos fueron; imipenem/cilastatina: 41.08 , piperacilina/ tazobactam 31.35, vancomicina con $30,75 \mathrm{DDD} / 100$ camas/día mostrando cifras superiores a las encontradas en nuestro estudio.

Se calcularon las DDD de los antimicrobianos por cada año de estudio en un hospital de 629 camas en ciudad de México y se encontró un consumo de ceftriaxona del 2013 al 2017, con un pico máximo en ese año de 24.8. En cuanto a ceftazidima y cefepime se registran disminuciones porcentuales de 44 y de 25 DDD. Con relación a la categoría de las quinolonas, el consumo de levofloxacina ha mostrado un incremento constante: pasó de 7.1 en 2013 a 11.3 en 2017; en el caso de ciprofloxacina, se ob- serva una disminución paulatina con una DDD en 2013 de 3.8 a 2.8 en el año 2018. En cuanto a la categoría de los carbapenémicos, es notable la reducción en el consumo de imipenem/cilastina, con una DDD en el 2013 de 2.3 que bajó a 0.7 en el 2018 [14]. Estos hallazgos se relacionan con lo observado en nuestro estudio.

En un estudio promovido por la Sociedad Chilena de Infectología relacionada con el consumo de antimicrobianos de 15 hospitales; se presenta la información de DDD por cada 100 días cama en los Servicios de Medicina y Cirugía mostrándose para cada antimicrobiano el promedio y rango de consumo. En Medicina la ceftriaxona es el antimicrobiano más consumido, seguido de cloxacilina; el consumo de ambos antimicrobianos es bastante dispar entre las distintas instituciones, lo que se ve reflejado en el amplio rango de consumo. Destaca además que el consumo de carbapenémicos es bastante similar entre las distintas instituciones, siendo imipenem el más consumido. Cuando vemos los antimicrobianos no b-lactámicos seleccionados (amikacina ciprofloxacina, clindamicina, metronidazol y vancomicina) los promedios de consumo de dichos antimicrobianos son similares [15]. El meropenem con 41.79 DDD/100 camas-día fue el antibiótico de reserva de mayor consumo en el servicio de medicina interna de un hospital en Perú durante un estudio de 6 meses en el año 2019, seguido de ciprofloxacina con 31.06 y metronidazol con 23.84, donde los autores destacan que es necesario mejorar las políticas dirigidas a la promoción del uso racional de antimicrobianos, evitando su resistencia y garantizando la optimización de los recursos [16].

\section{CONCLUSIONES}

La DDD emerge como un mejor sistema de cuantificación del consumo de antibióticos y otros medicamentos por lo que se debe institucionalizar su uso en nuestros hospitales para procurar un uso racional de los antibióticos.

Los mayores valores de DDD días cama observadas en los grupos farmacológicos de antibióticos como carbapenémicos, cefalosporinas de tercera generación, penicilina MRSA y quinolonas nos hace suponer la imperiosa necesidad de establecer controles reales sobre el uso de estos agentes antiinfecciosos. Por otra parte, los valores bajos de DDD de antibióticos como ceftazidima, cefoxitina, gentamicina, amikacina, e imipenem/cilastatin nos origina las obligadas preguntas: ¿Estos antibióticos se dejaron de usar?, ¿Ya no tienen un rol importante en la terapéutica intrahospitalaria?, ¿Preferimos usar medicamentos de mayor potencia como las cefalosporinas de tercera generación, quinolonas?

Adicionalmente la DDD en sus diversas modalidades, permite una mejor panorámica de su uso especialmente en los sistemas públicos de salud, donde los aspectos financieros son importantes para mantener un sistema de compras equilibrado. 
Recomendamos que los indicadores de Dosis Diaria Definida, Dosis Diaria Prescrita, así como Costo DDD sean usados rutinariamente en los servicios de farmacia en los diversos hospitales públicos del país y que los mismos sean estandarizados para obtener un valor real del consumo antimicrobiano.

El uso de notificaciones usadas por los SDMDU debe emplearse a cabalidad por parte de los farmacéuticos que visitan a diario las salas de hospitalización para captar la orden médica dada a los pacientes, como una manera de "alarma" ante el uso inadecuado de antibióticos.

Limitaciones del Estudio: hubiere sido prudente conocer el por qué se originó exactamente el uso del antibiótico en un paciente a través de los resultados de las pruebas de microbiología que hubieran podido enriquecer con precisión la prescripción de este. Debido al tiempo establecido para realizar el estudio y que la captura de la información se hacía después de las horas laborables de los farmacéuticos en sus hospitales no se pudo extender por más tiempo esta etapa del estudio. Así mismo, extender la aplicación de la DDP en los servicios quirúrgicos en los diversos hospitales.

Agradecimiento: a la Dirección General del Instituto Conmemorativo Gorgas de Estudios de la Salud por el apoyo ofrecido para realizar de esta investigación. Al Ministerio de Salud y a la Caja de Seguro Social por facilitar la participación de los farmacéuticos para la captura de la información del estudio, especialmente a la Lic. Gisela Tejada HSJ, Lic. René Rivera HSMA, Lic. Aurea González HRH, Lic. Xiomara Espinosa HLF.

\section{REFERENCIAS}

[1] Boletín: Perspectivas políticas sobre medicamentos de la OMS. Promoción del uso racional de medicamentos: componentes centrales. Septiembre de 2002, Ginebra, Suiza. Organización Mundial de la Salud.[2]Organización Mundial de la Salud, Resistencia a los antimicrobianos, Sitio Web, Fecha no disponible, URL: https://www.paho.org/es/temas/ resistencia-antimicrobianos

[3] Conte E, Morales Y, Moreno J, Herrera V, Zamorano C, Gómez B, Toro J: Estudio sobre resistencia bacteriana y uso racional de antibióticos en Panamá. Publicación del Instituto Conmemorativo Gorgas de Estudios de la Salud, Panamá 2016. ISBN 978-9962699-15-6.

[4] Girón N. D'alessio R. Sistema de distribución de medicamentos por dosis unitarias en Guía para el Desarrollo de Servicios Farmacéuticos Hospitalarios. Organización Panamericana de la Salud. Octubre 1997.

[5] Rodríguez C, Plata M, Agrázales G: Sistema de Distribución de Medicamentos en Dosis Unitaria. Dirección Nacional de Prestaciones Médicas, Caja de Seguro Social, Panamá 1994.
[6] Pascual-Salcedo M, Garjón F, Pina B, Labarta C: Estudio de utilización de medicamentos (Revisión de la literatura española) Revista Clínica Electrónica de Atención Primaria, España, 2003. https://ddd.uab.cat/ pub/rceap/rceap_a2008m2n15/ rceap_a2008m2n15a5.pdf

[7] Figueras A, Vallano A, Narváez E: Fundamentos metodológicos de los EUM, Una aproximación práctica para estudios en ámbito hospitalario. Fundación Institut Catalá de Farmacología. Universitat Autónoma de Barcelona. Hospital Universitari Vall d'Hebron. Barcelona, España, 2003.

[8] Rodríguez B, López J: Uso de antibióticos parenterales en el servicio de medicina interna de un hospital de tercer nivel de la ciudad de Bogotá, Rev. Colomb. Cienc. Quím. Farm. Vol. 38 (2), 142-155, 2009.

[9] Capellá D, La porte JR. Métodos aplicados en estudios descriptivos de utilización de medicamentos en Principios de Epidemiología del Medicamento. 2da edición, Barcelona, España, 1993.

[10] Rodríguez-Ganen O, Asbun-Bojalil J. Vigilancia del consumo de antimicrobianos en hospitales de México: situación actual y guía práctica para su implementación RevPanam Salud Publica vol.32 n.5 Washington nov. 2012. DOI: https://doi.org/10.1590/ S1020-49892012001100009

[11] Ávila F, Ruiz I, Jara M, Pizarro E.; Consumo de antibióticos de uso restringido y semirrestringido en un hospital público de alta complejidad" Tesis de Grado. Universidad de Chile, 2013.

[12] Arnao L, Celis J. Consumo, indicación y prescripción de antibióticos de reserva en los Servicios de Medicina Interna, Cirugía General y Cuidados Intensivos de Adultos del Hospital Nacional Edgardo Rebagliati Martins - Es Salud, Lima-Perú en el 2006.

[13] Jara Olivo K, Montalvo Jaramillo J.: "Estudio de utilización de antibióticos en el servicio de medicina interna y cirugía general del hospital San Francisco de Quito-IESS en los trimestres enero - marzo 2014 2015" http://www.dspace.uce.edu.ec/handle/ 25000/11451).

[14] Sosa-Hernández O, Vázquez-Zamora C, GutiérrezMuñoz VH, Lugo-Zamudio G, Cureño-Díaz M. Resultados del Programa de Uso Racional de Antimicrobianos en un hospital de México, 20132018. Rev. Panam Salud Pública. 2020;44: e45. https:// doi.org/10.26633/RPSP.2020.45. DOI: https:// doi.org/10.26633/RPSP.2020.45

[15] Domínguez Isabel, Rosales Ruth, Cabello Ángela, Bavestrello Luis y Labarca Jaime. Evaluación del consumo de antimicrobianos en 15 hospitales chilenos. Resultados de un trabajo colaborativo, 2013. Rev. Chilena Infectol 2016; 33 (3): 307-312. DOI: https://doi.org/10.4067/S071610182016000300010

[16] Herrera Lino M, Santa Cruz O. Consumo y gasto de antibióticos de reserva en pacientes hospitalizados de Medicina Interna Hospital Vitarte enero junio 2019. Lima, Perú 2019. https:// cybertesis.unmsm.edu.pe/handle/ 20.500.12672/11451 